\title{
EDITORIAL
}

\section{Hope: Concerning structure and function}

There is little convincing agreement in the literature on the definition, measurement, and application of hope. The usual platitude-where there's life, there's hope-does not clarify the confusion, and clinical practice puts paid to a simplistic approach to hope. A 28 year-old man with widespread melanoma is hoping the doctor will offer him a new treatment. He hopes he will marry and have a family. The hopes to us are unrealistic-but for him they are tangible, the stuff of life, the dreams that keep him going. What should clinicians do with this man's hopes? Should we support him medically, for the sake of hope? Does hoping prolong life against death? Should we encourage unproven therapies as elixirs of hope? When hope clashes with reality and causes angst, is it better to jettison hope, or reality? What does acceptance of death mean in terms of hope? What can it mean to live without hope?

There are many questions and so it is good to see in this issue of the journal two articles tackling hope.

Wahl et al. ("The Norwegian Version of the Herth Hope Index (HHI-N): A Psychometric Study") developed a Norwegian version of the Herth Hope Index (HHI-N) (which was originally developed in an illness cohort) and apply it to the general population. This study examines an important theoretical assumption, that the nature of hope is an universal concept and is applicable in health as in illness, in all cultures, and across the different stages of life. However the HHI-N has items such as "faith that comforts" and "give and receive caring/love," which many authors would not regard as core features of hope and could make universal application problematic.

In the second article, Nekolaichuk and Bruera ("Assessing Hope at the End of Life: Validation of an Experience of Hope Scale in Advanced Cancer Patients") apply the Hope Differential-Short (HDS) scale to patients with advanced cancer. The study finds a negative correlation between hope and de- pression, which confirms previous research. Further, the authors raise the vital idea of using the HDS to measure whether hope varies over time in a given patient, especially in a chronic illness. This is important given the known association between hopelessness and a desire for hastened death (Breitbart et al., 2000). Nekolaichuk and Bruera cautiously term the HDS an experience of hope scale with items including "honest," "tender," and "trustful," which are affective by nature. Some authors however define hope as primarily a cognitive construct and the affect associated with hope as an epiphenomenon (Gum \& Snyder, 2002).

These studies highlight the challenges of studying hope, particularly in palliative care. So what are our shortcomings?

I see two key interrelated problems. First, hope is still not adequately defined conceptually, in particular in application to the terminally ill. Second, there is a recurring tendency in the palliative care literature when developing scales to mistake hope's content and purpose for the mechanism of hope. The literature expresses hope variously as meaning, faith, trust, caring, and belief. While each of these factors may be a consequence or a supporting strut of hoping, each could just as well be a final outcome of other phenomena, such as religious identification. It is a bit like not distinguishing between the mode of transport (a hot-air balloon) and the journey's purpose (holiday) and destination (Tasmania). Hence the waters are muddied.

This is of practical importance. By distinguishing the mechanism from its manifestation we have a greater chance of rectifying a problem. A medical analogy might be that while opioids relieve pain, it is better to diagnose the cause and treat it. If we do not identify the core characteristics of hope we will not be able to study hope in its many applications.

Where the definition of hope becomes too inclusive it quickly becomes unclear what we are measuring. So, what should we measure? 
Snyder has defined the mechanism of hoping as a "perceived capability to produce workable routes to desired goals (pathways thinking) and the requisite motivation to use those routes (agency thinking)" (Gum \& Snyder, 2002 pp 884). He has developed it into a validated structure and applied it to many scenarios, including palliative care. Earlier, Nunn (1996) had written a very fine article wherein he characterizes the key features of hope in terms of time, desirability, and expectancy. He has also created a hopefulness scale. Both these definitions focus on the mechanisms of hoping and allow hope to be effectively manipulated, measured, and studied. Nevertheless neither has been tested by trial in the terminally ill, and conceivably a more refined theoretical structure and accompanying scale awaits development.

The value of studying hope in palliative care is to understand the role it plays in psychological health, in making decisions about treatment, and in establishing goals of care. It will enable us to converse with a patient about hope, just as we do about depression, and to know when and how to encourage hope or, to recognize when hope is causing harm and how to redirect it. At times it might be best to avoid hoping for the future and live just for the present. In other circumstances Lifton's (1973) concept of symbolic immortality-values that transcend death, such as family or a legacy of workoffers a secure exit strategy.

\section{REFERENCES}

Breitbart, W., Rosenfeld, B., Pessin, H., et al. (2000). Depression, hopelessness, and desire for hastened death in terminally ill patients with cancer. Journal of the American Medical Association, 284, 2907-2911.

Gum, A. \& Snyder, C.R. (2002). Coping with terminal illness: The role of hopeful thinking. Journal of Palliative Care, 5, 883-894.

Lifton, R.J. (1973). Twentieth Annual Karen Horney Lecture. The sense of immortality: On death and the continuity of life. American Journal of Psychoanalysis, $33,3-15$.

Nunn, K.P. (1996). Personal hopefulness: A conceptual review of the relevance of the perceived future to psychiatry. British Journal of Medical Psychology, 69, 227-245.
SIMON WEIN, M.D. Co-Editor 